\title{
New Type of Asymmetric Fission in Proton-Rich Nuclei
}

A. N. Andreyev, ${ }^{1,2}$ J. Elseviers, ${ }^{1}$ M. Huyse, ${ }^{1}$ P. Van Duppen, ${ }^{1}$ S. Antalic ${ }^{3}$ A. Barzakh, ${ }^{4}$ N. Bree,${ }^{1}$ T. E. Cocolios,${ }^{1}$ V. F. Comas, ${ }^{5}$ J. Diriken, ${ }^{1}$ D. Fedorov,${ }^{4}$ V. Fedosseev, ${ }^{6}$ S. Franchoo, ${ }^{7}$ J. A. Heredia, ${ }^{5}$ O. Ivanov, ${ }_{1}^{1}$ U. Köster, ${ }^{8}$ B. A. Marsh, ${ }^{6}$ K. Nishio, ${ }^{9}$ R. D. Page, ${ }^{10}$ N. Patronis, ${ }^{1,11}$ M. Seliverstov, ${ }^{1,4}$ I. Tsekhanovich, ${ }^{12,17}$ P. Van den Bergh, ${ }^{1}$ J. Van De Walle, ${ }^{6}$ M. Venhart, ${ }^{1,3}$ S. Vermote, ${ }^{13}$ M. Veselsky, ${ }^{14}$ C. Wagemans, ${ }^{13}$ T. Ichikawa, ${ }^{15}$ A. Iwamoto, ${ }^{9}$ P. Möller, ${ }^{16}$ and A. J. Sierk ${ }^{16}$

${ }^{1}$ Instituut voor Kern- en Stralingsfysica, K.U. Leuven, University of Leuven, B-3001 Leuven, Belgium

${ }^{2} S$ chool of Engineering, University of the West of Scotland,

Paisley, PA1 2BE, United Kingdom, and the Scottish Universities Physics Alliance (SUPA)

${ }^{3}$ Department of Nuclear Physics and Biophysics, Comenius University, Bratislava, SK-84248, Slovakia

${ }^{4}$ Petersburg Nuclear Physics Institute, 188350 Gatchina, Russia

${ }^{5}$ Gesellschaft für Schwerionenforschung (GSI), Planckstrasse 1, D-64291 Darmstadt, Germany

${ }^{6}$ ISOLDE, CERN, CH-1211 Geneve 23, Switzerland

${ }^{7}$ Institut de Physique Nucléaire, IN2P3-CNRS/Université Paris-Sud, F-91406 Orsay Cedex, France

${ }^{8}$ Institut Laue Langevin, 6 rue Jules Horowitz, F-38042 Grenoble Cedex 9, France

${ }^{9}$ Advanced Science Research Center, Japan Atomic Energy Agency (JAEA), Tokai-mura, Naka-gun, Ibaraki, 319-1195, Japan

${ }^{10}$ Department of Physics, Oliver Lodge Laboratory, University of Liverpool, Liverpool L69 7ZE, United Kingdom

${ }^{11}$ Department of Physics, University of Ioannina, GR-45110 Ioannina, Greece

${ }^{12}$ School of Physics and Astronomy, University of Manchester, Manchester, M13 9PL, United Kingdom

${ }^{13}$ Department of Physics and Astronomy, University of Gent, Proeftuinstraat 86, B-9000 Gent, Belgium

${ }^{14}$ Institute of Physics, Slovak Academy of Science, Bratislava, Slovakia

${ }^{15}$ Yukawa Institute for Theoretical Physics, Kyoto University, Kyoto 606-8502, Japan

${ }^{16}$ Theoretical Division, Los Alamos National Laboratory, Los Alamos, New Mexico 87545, USA

${ }^{17}$ Centre d'Etudes Nucleaires de Bordeaux Gradignan, F-33175 Gradignan Cedex, France

(Received 8 October 2010; published 14 December 2010)

A very exotic process of $\beta$-delayed fission of ${ }^{180} \mathrm{Tl}$ is studied in detail by using resonant laser ionization with subsequent mass separation at ISOLDE (CERN). In contrast to common expectations, the fissionfragment mass distribution of the post- $\beta$-decay daughter nucleus ${ }^{180} \mathrm{Hg}(N / Z=1.25)$ is asymmetric. This asymmetry is more surprising since a mass-symmetric split of this extremely neutron-deficient nucleus would lead to two ${ }^{90} \mathrm{Zr}$ fragments, with magic $N=50$ and semimagic $Z=40$. This is a new type of asymmetric fission, not caused by large shell effects related to fragment magic proton and neutron numbers, as observed in the actinide region. The newly measured branching ratio for $\beta$-delayed fission of ${ }^{180} \mathrm{Tl}$ is $3.6(7) \times 10^{-3} \%$, approximately 2 orders of magnitude larger than in an earlier study.

DOI: 10.1103/PhysRevLett.105.252502

PACS numbers: 24.75.+i, 23.40.-s, 27.70.+q

Nuclear fission, discovered more than 70 years ago [1], represents one of the most dramatic examples of a nuclear metamorphosis, whereby the nucleus splits into two fragments releasing a large amount of energy. Initially, the fission process was described within the liquid-drop model $[2,3]$, in which shape-dependent surface and Coulomb energy terms define the potential-energy landscape through which fission occurs. However, this macroscopic approach naturally leads to symmetric fragments and cannot explain observed asymmetric mass splits of actinides. Only by including a microscopic treatment based on shell effects can asymmetric fission be described [4]. Importantly, only in fission below or slightly above the barrier, so-called low-energy fission, can the interplay between the macroscopic liquid-drop contribution and the microscopic single-particle shell corrections be most fully explored.

Until recently, such low-energy fission studies were limited to nuclei from around thorium (Th) to fermium
(Fm) using spontaneous fission, fission induced by thermal neutrons or $\beta$-delayed fission. These studies showed the dominance of asymmetric fission over symmetric fission for most isotopes of these elements [5-7] and suggested that structure effects due to, specifically, the spherical shell structure of doubly magic ${ }^{132} \mathrm{Sn}$ dominate the mass split. A decade ago, a new technique, developed at GSI [8] - Coulomb-excited fission of radioactive beamsallowed for a more extensive experimental survey of lowenergy fission in other regions of the nuclidic chart. These studies demonstrated the transition from mostly asymmetric fission in the actinides towards symmetric fission as the dominant mode in the light thorium to astatine region. This is also consistent with earlier studies by Itkis et al. [9], in which fission of stable targets in the mass 185-210 region was induced by bombardment with protons and ${ }^{3,4} \mathrm{He}$ beams. Itkis et al. found mostly symmetric mass distributions in the region around ${ }^{208} \mathrm{~Pb}$, with about four systems in the mass $A \sim 200$ region having a slight reduction of 
probability for symmetric mass splits compared to those with about 5 fewer or more nucleons.

Another way to study low-energy fission is through $\beta$-delayed fission $(\beta \mathrm{DF})$, discovered in 1966 in Dubna for the isotopes ${ }^{232,234} \mathrm{Am}[10,11]$. In this two-step nuclear process, a parent nucleus first undergoes $\beta$ decay, in this case electron capture (EC), populating states in the daughter nucleus which then may fission, provided the energy release $Q_{\mathrm{EC}}$ of the parent nuclide is comparable to the fission-barrier height $B_{f}$ of the daughter nucleus. It is important to stress that the maximum excitation energy of the fissioning daughter nucleus is limited to the parent $Q_{\mathrm{EC}}$ value. $\beta$-delayed fission is of special interest because it allows the study of the low-energy fission properties of exotic nuclei possessing unusual neutron to proton ratios, e.g., $N / Z=1.25$ for ${ }^{180} \mathrm{Hg}$ studied in this work, in contrast to a typical ratio of $N / Z=1.55-1.59$ in the $\mathrm{U}$ region. Two areas in the nuclear chart were identified where $\beta \mathrm{DF}$ is experimentally accessible [12,13]: the $\mathrm{U}$ region (12 cases studied [14]) and a region near the very neutron-deficient lead $(\mathrm{Pb})$ isotopes.

The first experiments to measure $\beta \mathrm{DF}$ in the $\mathrm{Pb}$ region were performed two decades ago in Dubna, but the unselective production and identification methods used did not allow an unambiguous isotope assignment; the suggested $\beta \mathrm{DF}$ candidates were ${ }^{180} \mathrm{Tl},{ }^{196} \mathrm{At}$, and ${ }^{188} \mathrm{Bi}$ $[15,16]$. Half-life estimates for these three nuclides were obtained, and only for ${ }^{180} \mathrm{Tl}$ was the $\beta$-delayed fission probability $P_{\beta \mathrm{DF}}$, defined as the ratio between the $\beta \mathrm{DF}$ and the total $\beta$-decay probabilities, determined. An unexpectedly low value of $P_{\beta \mathrm{DF}}\left({ }^{180} \mathrm{Tl}\right)=3 \times 10^{-5 \pm 1} \%$ [16] was reported, which deviated by a factor of 100 from the known systematics in the $\mathrm{U}$ region (cf. [14]).

In this Letter, we report on a dedicated $\beta$ DF study of ${ }^{180} \mathrm{Tl}$ at the ISOLDE mass separator at CERN [17]. A $1.4 \mathrm{GeV}$ proton beam with an average intensity of $1.2 \mu \mathrm{A}$ impinges on a $50 \mathrm{~g} / \mathrm{cm}^{2} \mathrm{UC}_{x}$ target, producing a large variety of different nuclides. To obtain a high-purity source of ${ }^{180} \mathrm{Tl}$, allowing a precise study of its decay, a combination of resonance laser ionization and mass separation is used, resulting in a unique isotopic selection [18]. After selective ionization, acceleration up to $30 \mathrm{keV}$, and mass separation, a pure ${ }^{180} \mathrm{Tl}$ beam of $\sim 150$ atoms/s passes through a hole in an annular silicon detector and is implanted into a carbon foil of $20 \mu \mathrm{g} / \mathrm{cm}^{2}$ thickness; see Fig. 1. A second Si detector is placed $3 \mathrm{~mm}$ behind the foil. By using the two silicon detectors (both with a thickness of $300 \mu \mathrm{m}$ ), both single $\alpha$ and fission decays and double-fold fission-fragment coincidences can be measured. The total registration efficiency for a single $\alpha$ or fission decay in one of the $\mathrm{Si}$ detectors is $66 \%$, while coincident fission fragments are registered with an efficiency of $20 \%$. A segmented MINIBALL Ge cluster [19], consisting of three individual germanium crystals, and a planar Ge detector are installed surrounding the detection chamber to allow $\gamma$ and $K$ x-ray measurements in coincidence with particle

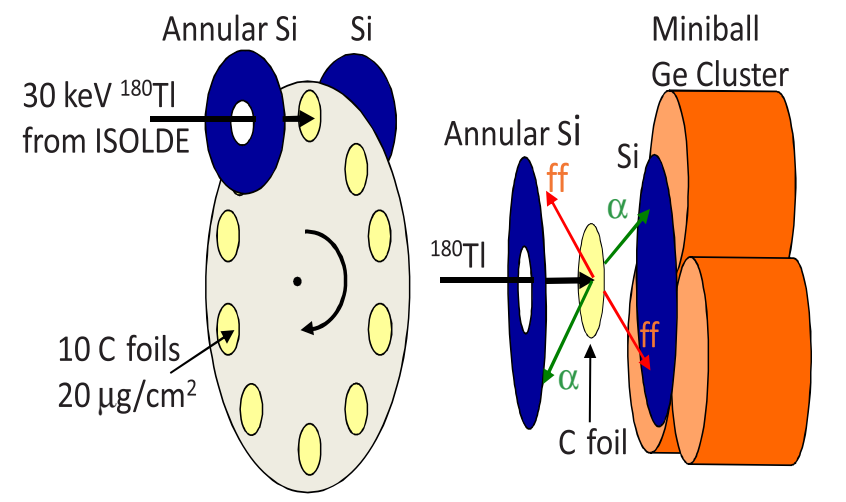

FIG. 1 (color online). A general view of the "windmill" setup used in the experiment-left side of the figure; a zoom of the detector arrangement is given on the right side.

events. In order to reduce the background from the longerlived daughter activities, ten carbon implantation foils on a wheel are used in a fixed implantation-decay mode.

In total, approximately $1.4 \times 10^{6} \alpha$ decays of ${ }^{180} \mathrm{Tl}$ are detected, and the $\alpha$-decay spectrum measured in two Si detectors is shown in Fig. 2(a). All observed $\alpha$ lines originate from ${ }^{180} \mathrm{Tl}$ or its subsequent decays (cf. the decay scheme in Fig. 3) as they are reduced by a factor of $\sim 70$ when laser light is blocked, preventing laser ionization of Tl. No direct production of ${ }^{180} \mathrm{Hg}$ is possible as it cannot be ionized either by surface ionization or by laser ionization tuned to $\mathrm{Tl}$ isotopes. This demonstrates the purity of the ${ }^{180} \mathrm{Tl}$ source, which allows an accurate determination of the different branching ratios. A half-life value of $T_{1 / 2}\left({ }^{180} \mathrm{Tl}\right)=1.09(1) \mathrm{s}$ is deduced, more precise than the literature value of 1.4(3) s [20]. The 1111 singles fission events in the region of $30-90 \mathrm{MeV}$ are observed when the lasers are tuned to $\mathrm{Tl}$ ionization and exhibit a half-life of
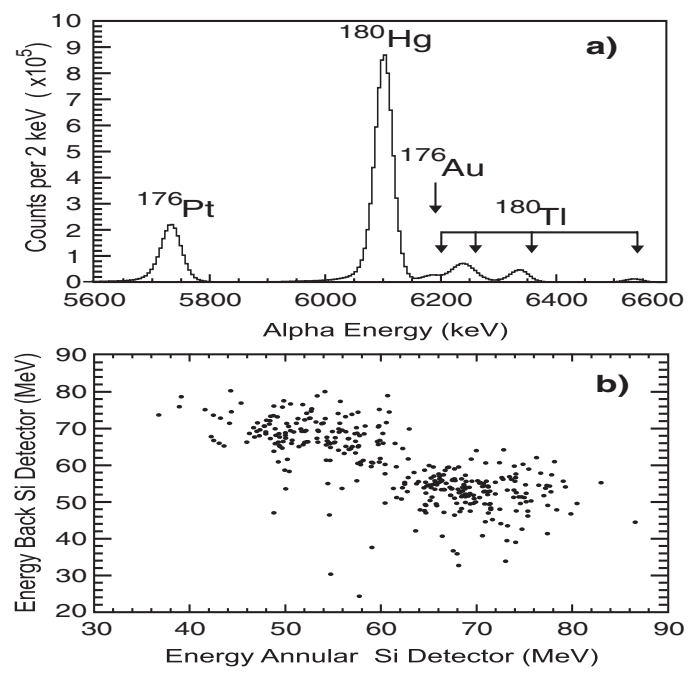

FIG. 2. (a) Singles $\alpha$-decay energy spectrum from both $\mathrm{Si}$ detectors; (b) Si-Si coincidence spectrum in the fission-energy region. The two-peaked structure in (b) originates because the two fission fragments have different energies, a direct result of the asymmetric mass distribution. 


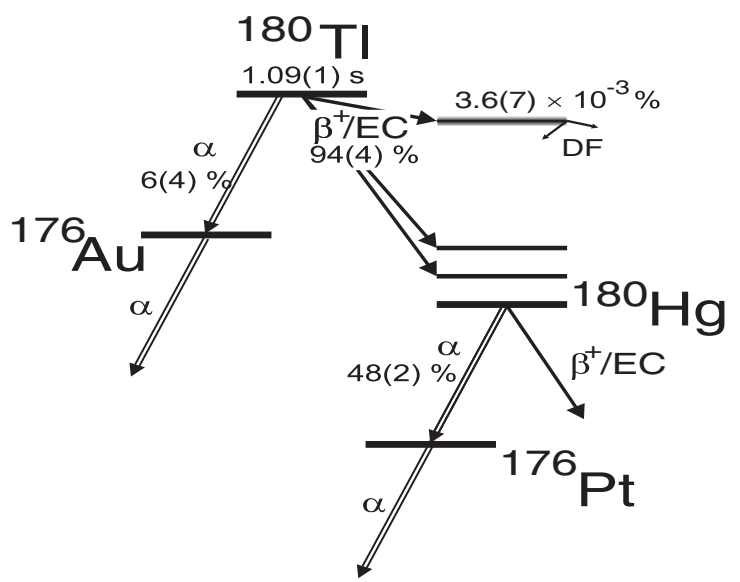

FIG. 3. A simplified decay scheme of ${ }^{180} \mathrm{Tl}$ with deduced halflife and branching ratios for its various decay modes. The $94(4) \% \beta^{+} / \mathrm{EC}$ decay branch of ${ }^{180} \mathrm{Tl}$ is shown schematically by arrows feeding excited states in ${ }^{180} \mathrm{Hg}$; those states in the vicinity of the fission barrier can undergo $\beta \mathrm{DF}$.

1.04(28) s. In total, 346 dual coincidences between fission fragments are observed; see Fig. 2(b). Finally, prompt coincidences between fission fragments and $\mathrm{Hg} K$ x rays are registered. These observations together unambiguously prove the observation of prompt fission of excited states in ${ }^{180} \mathrm{Hg}$ fed in the $\beta$ decay of ${ }^{180} \mathrm{Tl}$. This confirms that the fission events reported by Lazarev et al. [16] with a halflife of $0.97_{-0.07}^{+0.08} \mathrm{~s}$ indeed belong to the $\beta \mathrm{DF}$ of ${ }^{180} \mathrm{Tl}$.

The purity of the ${ }^{180} \mathrm{Tl}$ sample allows absolute branching ratios of its decay channels to be deduced by comparing the summed number of ${ }^{180} \mathrm{Tl} \beta$ decays and $\beta \mathrm{DF}$ events to the number of ${ }^{180} \mathrm{Hg} \alpha$ decays (see Figs. 2 and 3) using the well-known $\alpha$ branching ratio of ${ }^{180} \mathrm{Hg}$ [48(2)\% [21]] and a correction for the different half-lives due to the implantation-decay cycle. This results in an $\beta^{+} / \mathrm{EC}$ branching ratio of $94(4) \%$ and a $\beta \mathrm{DF}$ probability $P_{\beta \mathrm{DF}}\left({ }^{180} \mathrm{Tl}\right)=3.6(7) \times 10^{-3} \%$ for ${ }^{180} \mathrm{Tl}$. Thus, this work resolves long-standing questions about the experiments of Lazarev et al. [16]. They indeed observed $\beta \mathrm{DF}$, but, most probably due to an overestimation of the calculated production cross section of ${ }^{180} \mathrm{Tl}$, their reported $P_{\beta \mathrm{DF}}$ value was approximately 100 times too small. Our $P_{\beta \mathrm{DF}}$ value, using the calculated difference $Q_{\mathrm{EC}}\left({ }^{180} \mathrm{Tl}\right)-B_{f}\left({ }^{180} \mathrm{Hg}\right)=$ $0.63 \mathrm{MeV}$ from Ref. [13], is in agreement with the systematics of $P_{\beta \mathrm{DF}}$ as a function of $Q_{\mathrm{EC}}-B_{f}$ in the $\mathrm{U}$ region, the other region where $\beta \mathrm{DF}$ has been observed; see, e.g., [14].

A correct fission-fragment energy calibration using a ${ }^{252} \mathrm{Cf}$ source and taking pulse-height defects into account can be performed only for the 346 coincident fission events of ${ }^{180} \mathrm{Hg}$, and a fission-fragment mass distribution can be obtained through a well-established procedure $[22,23]$. The resulting spectrum as a function of the total kinetic energy and the fission-fragment mass is shown in Fig. 4. The mass distribution is clearly asymmetric, with the most probable heavy and light masses of $A_{H}=100(1)$ and $A_{L}=80(1)$, having a width of sigma $=4.0(3) \mathrm{amu}$. The most probable

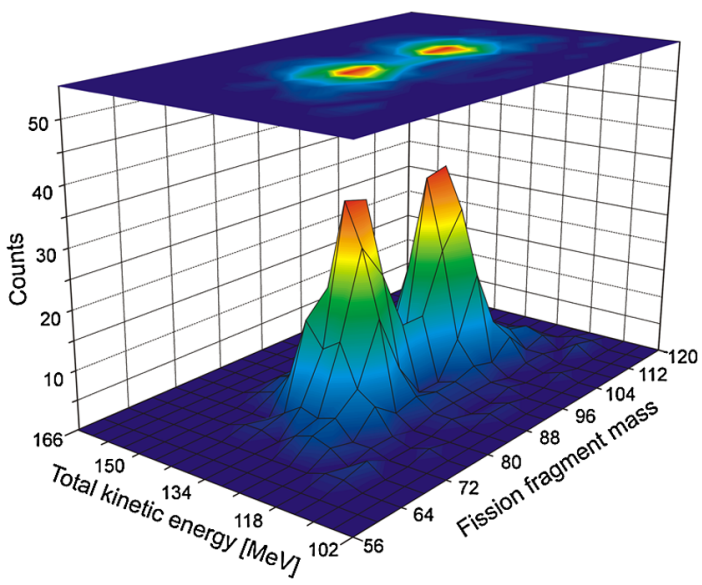

FIG. 4 (color online). The derived fission-fragment distribution of ${ }^{180} \mathrm{Hg}$ as a function of the fragment mass and the total kinetic energy.

$Z$ values of the heavy and light fission fragments are deduced to be $Z_{H}=44(2)$ and $Z_{L}=36(2)$, respectively, assuming that the $N / Z$ ratio of the parent nucleus ${ }^{180} \mathrm{Hg}$ is preserved in the fission fragments. Thus, the most abundantly produced fission fragments are ${ }^{100} \mathrm{Ru}$ and ${ }^{80} \mathrm{Kr}$ and their neighbors. Although $75 \%$ of the fission events are detected in coincidence with $\gamma$ rays, no discrete lines are observed due to Doppler broadening. The energy deposited in the $\mathrm{Ge}$ detectors reaches a maximum of $6.4 \mathrm{MeV}$. The average total kinetic energy as obtained from Fig. 4 is 134.6(7) $\mathrm{MeV}$ with a width of sigma $=5.6(8) \mathrm{MeV}$. The energy released in the $\beta \mathrm{DF}$ process is shared among the $\beta^{+} /$EC decay, the fragments (excitation and kinetic energy), and possible neutron emission. Assuming the fragments ${ }^{100} \mathrm{Ru}$ and ${ }^{80} \mathrm{Kr}$, this energy release is $\Delta M\left({ }^{180} \mathrm{Tl}\right)-\Delta M\left({ }^{100} \mathrm{Ru}\right)-\Delta M\left({ }^{80} \mathrm{Kr}\right)=157.8 \mathrm{MeV}$ [24]. Then, by accounting for the energy released in $\gamma$ emission, we find that only the evaporation of at most one neutron is energetically possible. It is important to stress that the maximum excitation energy of ${ }^{180} \mathrm{Hg}$ is limited to the parent $Q_{\mathrm{EC}}\left({ }^{180} \mathrm{Tl}\right)=10.44 \mathrm{MeV}$ [13].

The most surprising result of this study is the asymmetric mass distribution of the fission fragments of ${ }^{180} \mathrm{Hg}$. Indeed, one might have expected a symmetric fissionfragment mass distribution, as this was observed to be the main mode of the low-energy fission in a broad neutron-deficient region below Th [8]. In addition, the explanations offered for the much smaller "apparent" asymmetry seen in the work of Itkis et al. [9,25] lead to a prediction that mass distributions will also be symmetric below $A \leq 195$. Finally, the very common arguments, applied, e.g., for the fission of heavy actinides, that fragment shell effects (rather than shell effects in the region near the saddle) determine mass distributions would also lead to an expectation of symmetry. This is because there are no strong ground-state shell effects in the measured asymmetric fragments of ${ }^{180} \mathrm{Hg}$, while the weak shell effect for the nucleus ${ }^{90} \mathrm{Zr}$, with magic $N=50$ and semimagic 


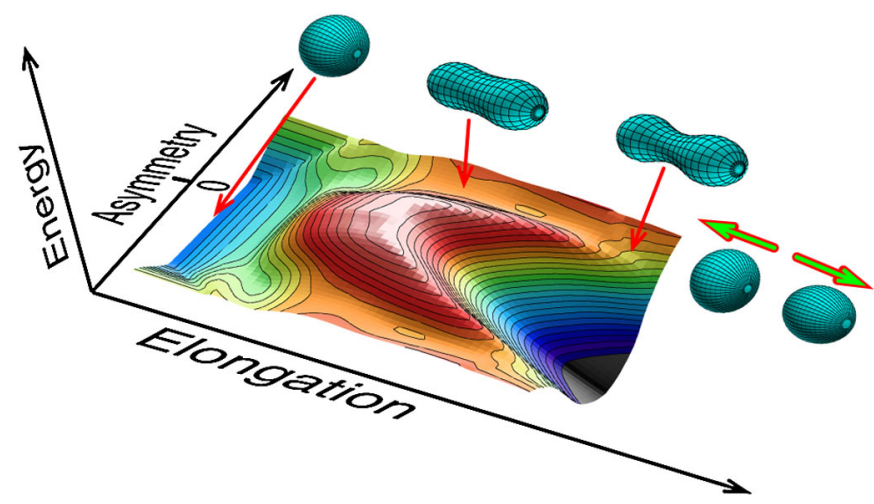

FIG. 5 (color online). A schematic representation of the potential-energy surface for ${ }^{180} \mathrm{Hg}$ in two dimensions (elongation and asymmetry) resulting from a five-dimensional analysis. The shapes shown, connected by arrows to their locations, are the ground state, the saddle point, and the point where the asymmetric valley disappears.

$Z=40$ would, if it were determinative, lead to symmetric splitting.

A realistic description of the fission process needs the structure of the full, multidimensional fission potentialenergy surface based on at least five independent shape parameters [26]. We use such a model to calculate the 5D fission potential-energy landscape for ${ }^{180} \mathrm{Hg}$ and employ the immersion method [13] to characterize the structure of this surface. In Fig. 5, the potential-energy surface in a twodimensional subspace of the full $5 \mathrm{D}$ deformation space is shown. The mass-symmetric ground-state minimum is given at the left of the figure. The only saddle exiting out of this minimum leads to the upper mass-asymmetric valley (or its reflected equivalent at the bottom). The saddle is calculated at $0.63 \mathrm{MeV}$ below the $Q_{\mathrm{EC}}\left({ }^{180} \mathrm{Tl}\right)$ value [13]. There is another, mass-symmetric valley with energy below the asymmetric valley at larger elongations; they are separated from each other by a ridge, and the entrance to this valley lies at a much higher excitation energy; therefore, the system must pass over the asymmetric saddle point to fission. Starting from the saddle and following the asymmetric valley in the direction of increasing elongation, the ridge eventually disappears, and one might expect the system to then drop into the symmetric valley. But at this point the nuclear shape has a well-developed (small-radius) neck, which restricts the flow of matter so little mass flow can take place at this late stage of the division process prior to the disappearance of the neck. A dynamical model would be required to make a specific prediction of the mass split implied by our potential-energy calculations. Presently, we assume the mass asymmetry is frozen when the ridge disappears, which gives an upper limit on the predicted asymmetry. In this approach, the calculated mass asymmetry is $A_{H} / A_{L}=108 / 72$, which is reasonably consistent with the measured values.

In the actinide region, one can identify a connection between the asymmetric fission-fragment distribution and strong shell effects in the region near the saddle, extending and increasing as one moves toward the scission point. These shell effects are related to the extra binding energy of doubly magic ${ }^{132} \mathrm{Sn}$ (e.g., [7]). In contrast, in the neutron-deficient $\mathrm{Hg}$ region such a combined effect of magic proton and neutron shells in the observed fission fragments is completely absent, and the asymmetric split is determined by relatively small microscopic effects that do not persist to scission but which cause the fission saddle point and a nearby valley to be mass-asymmetric.

This new mode, which should survive only in very lowenergy fission, arises from the complex interplay between macroscopic and microscopic contributions to the total energy as a function of shape and is observed here for the first time. Predicting this mass asymmetry and related properties provides a stringent test for any nuclear structure model. Importantly, our model predicts asymmetric mass splits in a broader range of even-even isotopes ${ }^{176-196} \mathrm{Hg}$. Some of these nuclei can be studied through $\beta$-delayed fission, but other techniques such as fusion-fission experiments or fission of radioactive ions induced by Coulomb excitation [8] could also be used for detailed studies of this new phenomenon, provided they probe the region close to the fission barrier. It is a challenge to these experimental approaches as well as current theories to elucidate this new fission mode which gives the opportunity to study the topography and dynamics of the saddle to scission region, the least understood part of the fission process.

We thank the ISOLDE Collaboration for providing excellent beams and the GSI Target Group for manufacturing the carbon foils. This work was supported by FWOVlaanderen (Belgium), by GOA/2004/03 (BOF-K.U. Leuven), by the IUAP-Belgian State Belgian Science Policy (BriX network P6/23), by the European Commission within the Sixth Framework Program through I3-EURONS (Contract No. RII3-CT-2004-506065), by the United Kingdom Science and Technology Facilities Council (STFC), and by the Slovak grant agency VEGA (Contract No. 1/0091/10). This work was carried out under the auspices of the NNSA of the U.S. DOE at Los Alamos National Laboratory under Contract No. DE-AC5206NA25396 and a travel grant for P. M. to JUSTIPEN at RIKEN under Grant No. DE-FG02-06ER41407 (University of Tennessee). The work of T. I. was done in the Yukawa International Project for Quark-Hadron Sciences (YIPQS).

[1] O. Hahn and F. Strassmann, Naturwissenschaften 27 1, 11 (1939).

[2] N. Bohr and J. A. Wheeler, Phys. Rev. 56, 426 (1939).

[3] L. Meitner and O. R. Frisch, Nature (London) 143, 239 (1939).

[4] P. Möller and S. G. Nilsson, Phys. Lett. 31B, 283 (1970).

[5] F. Gönnenwein, in Nuclear Fission Process, edited by C. Wagemans (CRC Press, Boca Raton, FL, 1991). 
[6] H. L. Hall and D. C. Hoffman, J. Radiol. Nucl. Chem. 142, 53 (1990).

[7] Yu. Ts. Oganessian, J. Phys. G 34, R165 (2007).

[8] K.-H. Schmidt et al., Nucl. Phys. A693, 169 (2001); A665, 221 (2000).

[9] M. G. Itkis et al., Sov. J. Nucl. Phys. 52, 601 (1990); 53, 757 (1991).

[10] V. I. Kuznetzov and N. K. Skobelev, Yad. Fiz. 4, 279 (1966); 5, 271 (1967); 5, 1136 (1967); Fiz. Elem. Chastits At. Yadra 30, 1514 (1999).

[11] H. L. Hall and D. C. Hoffman, Annu. Rev. Nucl. Part. Sci. 42, 147 (1992).

[12] E. Ye. Berlovich and Yu. N. Novikov, Phys. Lett. 29B, 155 (1969).

[13] P. Möller et al., Phys. Rev. C 79, 064304 (2009).

[14] D. A. Shaughnessy et al., Phys. Rev. C 65, 024612 (2002).

[15] Yu. A. Lazarev et al., Europhys. Lett. 4, 893 (1987).
[16] Yu. A. Lazarev et al., Inst. Phys. Conf. Ser. N132, 739 (1992).

[17] E. Kugler, Hyperfine Interact. 129, 23 (2000).

[18] U. Köster et al., Nucl. Instrum. Methods Phys. Res., Sect. B 204, 347 (2003).

[19] J. Eberth et al., Prog. Part. Nucl. Phys. 46, 389 (2001).

[20] K. S. Toth et al., Phys. Rev. C 58, 1310 (1998).

[21] K. S. Toth et al., Phys. Rev. C 60, 011302 (1999).

[22] H. W. Schmitt, W.E. Kiker, and C. W. Williams, Phys. Rev. 137, B837 (1965).

[23] E. Weissenberger et al., Nucl. Instrum. Methods Phys. Res., Sect. A 248, 506 (1986).

[24] G. Audi, A. H. Wapstra, and C. Thibault, Nucl. Phys. A729, 337 (2003).

[25] S. I. Mulgin et al., Nucl. Phys. A640, 375 (1998).

[26] P. Möller et al., Nature (London) 409, 785 (2001). 